SHORT REPORT

\title{
Psychiatric disorders in inpatients on a neurology ward: estimate of prevalence and usefulness of screening questionnaires
}

\author{
Kate Jefferies, Arthur Owino, Hugh Rickards, Niruj Agrawal
}

Background: Patients on neurology wards have been shown to have high rates of psychiatric illness. Prevalence figures of 39$64 \%$ have been reported previously. However a low rate of recognition of psychiatric illness is also observed in this population.

Objectives: To estimate the prevalence of psychiatric illness in neurology inpatients in a regional neuroscience centre and to assess the sensitivity and specificity of a batch of screening questionnaires.

Method: Patients were assessed using the following screening questionnaires: Primary Care Evaluation of Mental Disorders, Mini Mental State Examination, Frontal Assessment Battery, Alcohol Use Disorders Identification Test and a neurologistrating scale of organicity. All patients also had a full psychiatric assessment using the Diagnostic and statistical manual of mental disorders, 4th edition (DSM-IV). The screening questionnaires were then compared with our "gold standard", the psychiatric assessment.

Results: The prevalence of psychiatric illness (as determined by the psychiatric interview) in neurology inpatients in a tertiary referral centre was found to be $51.3 \%$ (95\% confidence interval 44 to $58 \%$ ). The sensitivity of this batch of screening questionnaires is $81.2 \%$ and the specificity is $77.1 \%$.

Conclusion: A high prevalence of psychiatric disorder was observed in inpatients on a dedicated neurology ward. The screening questionnaires used had a high sensitivity and specificity and could therefore be used as a simple way of identifying those with psychiatric illness.

$\mathrm{H}$ igh levels of psychiatric illness have been reported in general neurology inpatients and outpatients. Fink et al ${ }^{1}$ found a prevalence of $55.1 \%$ for current mental disorder in new neurological patients. Carson et $\mathrm{al}^{2}$ reported a prevalence of $47 \%$ for anxiety and depressive disorders in patients referred to general neurology outpatient clinics. Bridges and Goldberg ${ }^{3}$ showed the prevalence of psychiatric illness in neurology inpatients to be $39 \%$. Carson et al ${ }^{4}$ reported that $30 \%$ of patients referred to a neurologist had symptoms not explained by organic disease. A comparison of neurology outpatients with and without emotional disorders has shown that physical function, physical role function, bodily pain and social functioning are worse in those with emotional disorders. ${ }^{2}$

It has also been shown that psychiatric illnesses are commonly missed in neurological patients. Bridges and Goldberg ${ }^{3}$ showed that $72 \%$ of psychiatric illness was unrecognised by neurologists, and Fink et al found that only $1.5 \%$ of the patients with a mental illness were referred to a psychiatrist.
Carson et $a l^{2}$ found that only $11 \%$ of neurology patients with an emotional disorder expressed any perceived need for psychiatric input, whereas Bridges and Goldberg ${ }^{3}$ reported that $58 \%$ of neurology patients with a psychiatric illness, and $50 \%$ of those without, wished that an enquiry into their mood had been made.

To date, no study has investigated the prevalence rates of mental illness in neurology inpatients admitted to a regional neurosciences centre. It cannot be assumed that the rates would be the same as those reported for neurology outpatients.

The aims of this study were to:

- Estimate the prevalence of psychiatric disorder in neurology inpatients in a regional neuroscience centre

- Identify any high-risk groups

- Assess the effectiveness of a batch of screening questionnaires in the identification of mental illness.

\section{METHODS}

All new admissions to a neurology ward during a 6-month period were seen for screening and a psychiatric assessment. The only patients excluded were repeat admissions and private patients. Screening was undertaken using the following questionnaires:

- The anxiety and depression modules of the Primary Care Evaluation of Mental Disorders ${ }^{5}$

- Mini-Mental State Examination

- The Frontal Assessment Battery ${ }^{6}$

- Alcohol Use Disorders Identification Test questionnaire ${ }^{7}$

- A neurologist rating scale of "organicity"4

The screening questionnaires were used as a "batch". If patients scored above the threshold on one or more of the questionnaires, they were deemed to have a positive result on screening.

All patients had a clinical interview ("the gold standard") by the same clinician who carried out the screening. If relevant, diagnosis according to the Diagnostic and statistical manual of mental disorders, 4th edition (DSMIV)were assigned. Age, sex and neurological diagnosis were also recorded, so we could calculate whether certain groups of patients were more likely to require psychiatric attention.

Treatment recommendations were made for patients found to have a mental illness.

Abbreviations: DSM-IV, Diagnostic and Statistical Manual of Mental Disorders, 4th edition 


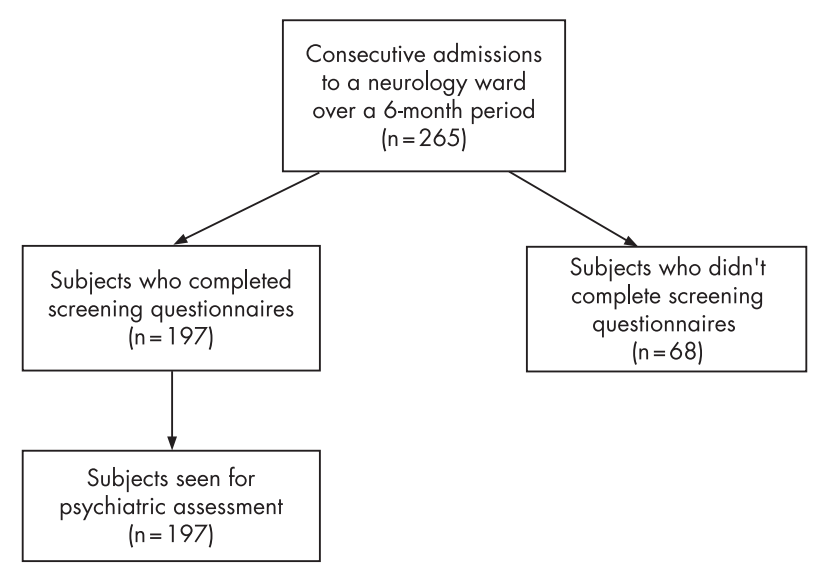

Figure 1 Algorithm for screening process.

\section{RESULTS}

\section{Characteristics of the sample}

There were 265 consecutive admissions during the study period (fig 1). In total 197 (74\%) patients were seen by the researchers; $57.4 \%$ were women and $42.6 \%$ were men. The age range was 18-91 years (mean (SD) 54.5 years). In all, $56 \%$ of patients had a known neurological diagnosis, and $44 \%$ had been admitted for diagnostic investigations.

\section{Prevalence of mental disorder in our sample}

In total, $51.3 \%$ of patients fulfilled the DSM-IV criteria for diagnosis of one or more mental disorders (95\% confidence interval $44 \%$ to $58 \%$ ), $18.7 \%$ fulfilled the criteria for two diagnoses and $5.1 \%$ fulfilled the criteria for three or more diagnoses (table 1).

\section{Risk factors for mental illness}

There were no differences in rates of mental illness between groups in terms of age, sex, or based on whether subjects had a known neurological diagnosis.

\section{Are the screening questionnaires correctly identifying the people with mental illness?}

Presence of a DSM-IV diagnosis on the Primary Care Evaluation of Mental Disorders was taken as a positive result. A cut-off of $\leqslant 26$ was used for the Mini Mental State Examination and $\geqslant 8$ for the Alcohol Use Disorders Identification Test questionnaire. As there was a high correlation between the Mini Mental State Examination and the Frontal Assessment Battery in detection of caseness (correlation coefficient $=0.776)$, the Frontal

Table 1 Rates of mental illness

\begin{tabular}{ll}
\hline DSM-IV diagnosis & $\begin{array}{l}\text { Rate in } \\
\text { subjects (\%) }\end{array}$ \\
\hline Mood disorders & 24.8 \\
Delirium, dementia and cognitive disorders & 17.7 \\
Anxiety disorders & 12.7 \\
Adjustment disorders & 4.6 \\
Somatoform disorders & 4.5 \\
Substance use disorders & 3 \\
Personality disorders & 2 \\
Disorders usually diagnosed in childhood & 2 \\
Other disorders that may be of clinical importance & 2 \\
Psychotic disorders & 1 \\
Eating disorders & 0.5 \\
\hline
\end{tabular}

DSM-IV, Diagnostic and Statistical Manual of Mental Disorders, 4th edition.
Assessment Battery was not used in the final analysis of the sensitivity and specificity.

Of the 197 patients seen, 104 scored positively on screening. Of the 101 who had a diagnosis of mental illness, 82 were picked up on screening and 19 were missed. The screening questionnaires falsely recognised 22 respondents as having mental illness.

Therefore, the sensitivity of the questionnaires as a whole is $81.2 \%(71.9-88 \%)$ and the specificity is $77.1 \%(67.2-84.8 \%)$.

There was no difference in age, sex, and neurological or psychiatric diagnosis for the 19 patients who had a diagnosis of mental illness but were missed by screening compared with the rest of the sample. Of the 19 patients who were missed by screening, only 12 had completed the full batch of questionnaires, whereas six had not been able to complete any of them.

\section{DISCUSSION}

In this study, we have shown that there are high prevalence rates of mental illness in neurology inpatients. These rates are similar to those previously found in neurology outpatients (47$64.6 \%),{ }^{12}$ but higher than the rates reported in neurology inpatients in generic wards $(39 \%) .{ }^{13}$

We found similar rates of anxiety and depression as previous researchers, but the prevalence of certain groups of psychiatric diagnoses differed in our group compared with those previously studied. Carson $e t a l^{4}$ have previously shown prevalence rates of non-organic disease to be as high as $30 \%$ in a general neurology outpatient clinic. We found the prevalence rate of somatoform disorders to be only $4.5 \%$. This can be explained by a number of factors. Some patients were assessed before an organic cause for their symptoms had been excluded. The prevalence rates for somatoform disorders may differ between inpatients and outpatients, or there may be variation in clinical practices between centres.

We showed a low rate of substance misuse in neurology inpatients (3\%). Previous prevalence studies looking at patients in a general hospital show rates of alcohol misuse or dependence to be as high as $30 \%$ in men and $8 \%$ in women. ${ }^{8}$

The batch of screening questionnaires used was shown to have a high sensitivity (81\%) and specificity (77\%). However, 19 people who had a diagnosis of mental illness were missed by screening. Six of the $19(32 \%)$ patients missed by the screening procedure had been unable to complete any of the screening questionnaires. Only nine of the remaining $182(5 \%)$ patients had not completed any of the questionnaires. If only those who were able to complete the full screening examination were studied, the sensitivity and specificity would be more favourable. The group of patients who were unable to complete any of the questionnaires have a high rate of neuropsychiatric illness. Six of the $15(40 \%)$ patients who did not complete any of the screening questionnaires were found to have mental illness after psychiatric assessment. When instituting a screening protocol in clinical practice, it will be important to ensure that this group is not forgotten.

We saw a high proportion (74\%) of all the patients admitted during a 6-month period. However, patients who were missed by the researchers were typically those who were admitted for shorter periods of time. It is not clear whether these patients were significantly different from the rest of the study population and whether this had an effect on our reported prevalence figures.

The fact that the same researcher carried out both the screening questionnaires and clinical assessment might have introduced bias. However, our prevalence figures were in line with previous research. This maximised the number of patients who completed both components of the assessment and minimised inconvenience for patients. Patients were seen at 
various times during their admission. It would be useful in future research to follow up on the outcome of the admission so that a longitudinal view could be applied.

The screening and psychiatric assessments were well received by both patients and also the neurology staff. No patients refused to be seen. Many made positive comments about the improved service. Later survey of the neurologists showed that they found this service useful and acceptable, and were keen for it to be continued.

\section{CONCLUSIONS}

There is a high prevalence of psychiatric disorder in inpatients on a dedicated neurology ward. The screening questionnaires used had a high sensitivity and specificity and could therefore be used as a simple way of identifying those with psychiatric illness. Considering the high rate of undetected psychiatric comorbidity in this population, which results in distress and burden, this screening process may offer a cost-effective solution that is acceptable to patients and clinicians.

\section{ACKNOWLEDGEMENTS}

We thank Dr Alan Carson for his advice on screening instruments, Dr Sarah White for her assistance with the statistical analysis and all the staff on Kent Ward for their help in completing the screening questionnaires.

\section{Authors' affiliations}

Kate Jefferies, Farnham Road Hospital, Guildford, Surrey, UK

Arthur Owino, Shaftsbury Clinic, Springfield University Hospital, London, UK

Hugh Rickards, Department of Neuropsychiatry, Queen Elizabeth Psychiatric Hospital, Edgbaston, Birmingham, UK
Niruj Agrawal, Neuropsychiatry Service, Clare House, St George's Hospital, London, UK

Competing interests: None.

Correspondence to: Dr K Jefferies, Farnham Road Hospital, Guildford, Surrey GU2 7LX, UK; katejefferies@tiscali.co.uk

Received 25 July 2006

Revised 10 October 2006

Accepted 14 October 2006

Published Online First 20 October 2006

\section{REFERENCES}

1 Fink $\mathbf{P}$, Hansen $M$, Sondergaard $L$, et al. Mental illness in new neurological patients. J Neurol Neurosurg Psychiatry 2003;74:817-19.

2 Carson A, Ringbauer B, MacKenzie L, et al. Neurological disease, emotional disorder, and disability: they are related: a study of 300 consecutive new referrals to a neurology outpatient department. I Neurol Neurosurg Psychiatry 2000;68:202-6.

3 Bridges K, Goldberg D. Psychiatric illness in inpatients with neurological disorders: patients' views on discussion of emotional problems with neurologists. BMJ 1984;289:656-8.

4 Carson A, Ringbaver B, Stone J, et al. Do medically unexplained symptoms matter? A prospective cohort study of 300 new referrals to neurology outpatient clinics. J Neurol Neurosurg Psychiatry 2000;68:207-10.

5 Spitzer RL, Williams JBW, Kroenke K, et al. Utility of a new procedure for diagnosing mental disorders in primary care; The PRIME-MD 1000 Study. JAMA 1994;272:1749-56.

6 Dubois B, Slachersky A, Litvan I, et al. The FAB, a frontal assessment battery at bedside. Neurology 2000;55:1621-6.

7 Saunders JB, Aasland OG, Babor TF, et al. Development of the alcohol use disorders identification test (AUDIT): WHO collaborative project on early detection of persons with harmful alcohol consumption-II. Addiction 1993;88:791-803.

8 Hearne R, Connolly A, Sheehan J. Alcohol abuse: prevalence and detection in a general hospital. J R Soc Med 2002;95:84-7. 


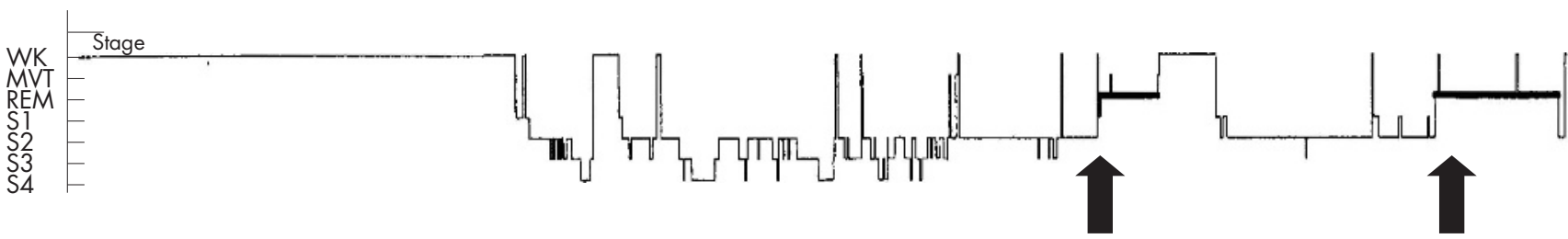

Figure 1 Sleep staging from the polysomnogram is shown, with wake (WK), movement (MVT), rapid eye movement (REM) sleep and the non-REM sleep stages, S1, S2, S3 and S4. There was some delay before the patient fell asleep but the overall sleep architecture was then normal with two episodes of abnormal movements during REM highlighted with the black arrows. The patient was in bed from 23:00 to 06:00 and the two events occurred at 02:15 and 04:30.

of other patients with REM abnormalities, ${ }^{34}$ suggesting that the variable developmental, pontine disorder in Möbius syndrome can cause REM disturbance if the critical neural networks within the pedunculopontine nucleus are affected. Secondary causes of RBD with structural pontine lesions support this theory. ${ }^{5}$ It is also possible that the condition is underreported or underdiagnosed and that more cases will emerge as RBD becomes more widely recognised.

The description of the stereotyped disturbing dreams with dream enactment in the absence of other causes of sleep disturbance on the polysomnography is characteristic of RBD, but atypical features include the early age of onset and treatment resistance. The young age of onset could be explained by a developmental rather than a neurodegenerative disorder. The reason for the lack of response to treatment remains unclear.

The case is unusual but highlights the need to question patients with Möbius syndrome specifically about sleep disturbance and it supports the theory that Möbius syndrome is a variable disorder affecting brainstem development.

Kirstie Anderson, John Shneerson, lan Smith

Respiratory Support and Sleep Centre, Papworth Hospital, Papworth Everard, Cambridge, UK

Correspondence to: Kirstie Anderson, Respiratory Support and Sleep Centre, Papworth Hospital, Papworth Everard, Cambridge CB3 8RE, UK ; kirstie.anderson@ntlworld.com

doi: 10.1136/jnnp.2006.105494

\section{References}

1 Verzijl HT, van der Zwaag B, Cruysberg JR, et al. Mobius syndrome redefined: a syndrome of rhombencephalic maldevelopment. Neurology 2003:61:327-33

2 Schenck CH. Mahowald MW. REM sleep behavior disorder: clinical, developmental, and neuroscience perspectives 16 years after its formal identification. Sleep 2002;25:120-38.

3 Mayer G, Blanke J. Moebius syndrome, narcolepsy and parasomnias: Report on two patients. Somnolgie 2004;8:110.

4 Parkes JD. Genetic factors in human sleep disorders with special reference to Norrie disease, PraderWilli syndrome and Moebius syndrome. J Sleep Res 1999;8(Suppl 1):14-22.

5 Plazzi G, Montagna P. Remitting REM sleep behavior disorder as the initial sign of multiple sclerosis. Sleep Med 2002;3:437-9.

\section{BOOK REVIEW}

\section{9/11 Mental health in the wake of terrorist attacks}

Edited by Yuval Neria, Ezra Gross, Randall Marshall. Cambridge University Press, Cambridge, 2006, $£ 60.00$ (hardcover), pp 630 . ISBN 0521831911

This book comprehensively describes the psychological response to the 9/1 terrorist attacks in New York and, to a lesser degree Washington DC. The impact of what happened on the local and US national population is considered through various epidemiological studies, as well as personal accounts from some of those more directly involved. These complement each other well and highlight the fact that some individuals developed marked psychological difficulties as a result of the attacks but the majority of those exposed have not. The results, particularly of Galea and colleagues' excellent work, suggest marked resilience among those involved. These find ings and increasing research evidence regarding the lack of effectiveness of universal interventions raise questions about some of the outreach and intervention work described in one section of the book.

There is clearly a need to provide practical and pragmatic support in an empathic manner following traumatic events and this appears to have been effectively done in many instance but questions remain unanswered regarding if, when and how more formal intervention should be provided. The absence of methodologically robust evaluation research concerning the programmes adopted suggests that an opportunity has probably been missed to further assess what best to do following traumatic events. Without doubt, many of those involved appear to have welcomed the support they received, but it is impossible to determine whether or not the interventions were truly effective in reducing levels of short term mental distress or the development of longer term psychological sequelae. These issues are discussed in more detail in the chapters of Litz, Shelev and Wesseley, which provide more critical commentaries on the approaches taken with full acknowledgement of the difficulty in deciding how best to respond.

The interesting chapters regarding community responses and effective leadership in extreme crisis support recent guidelines that suggest a move towards a more sociological approach to encourage resilience and support recovery. There are useful examples of the adoption of a stepped/stratified care approach and the provision of training and support for local service providers to provide specific interventions that have been shown to be effective, such as prolonged exposure treatment for post-traumatic stress disorder.

The chapter authors include a good mixture of recognised experts in the field and individuals who were directly involved and have developed an expertise as a result of this particular experience. A major strength of the book is the self-critical approach that many of the authors have taken when writing their chapters. They carefully discuss methodological issues and dilemmas that are likely to face anybody trying to provide services and conduct research in this area.

This book will undoubtedly serve as a very helpful resource for anybody who wants to learn more about the psychological aftermath and response to the $9 / 11$ terrorist attacks and should be used to inform the planning of psychosocial responses in the future.

Jonathan I Bisson

\section{CORRECTION}

doi: 10.1136/jnnp.2006.109058corr 1

J Moriarty. Psychiatric disorders in neurology patients. $J$ Neurol Neurosurg Psychiatry 2007;78:331. In the text and in the references of this paper the first author of its companion paper has been spelt incorrectly. The correct reference is Jefferies K, Owino A, Rickards H, et al. Psychiatric disorders in neurology inpatients: estimate of prevalance and usefulness of screening questionnaires. J Neurol Neurosurg Psychiatry 2007;78:414-16. 